\title{
Search for singly charged Higgs bosons in vector-boson scattering at ep colliders
}

\author{
Georges Azuelos, ${ }^{1,2, \dagger}$ Hao Sun, ${ }^{3, \$}$ and Kechen Wang ${ }^{4,5, *}$ \\ ${ }^{1}$ Université de Montréal, Montréal H3C 3J7, Canada \\ ${ }^{2}$ TRIUMF, Vancouver V6T 2A3, Canada \\ ${ }^{3}$ Institute of Theoretical Physics, School of Physics, Dalian University of Technology, \\ No. 2 Linggong Road, Dalian, Liaoning 116024, China \\ ${ }^{4}$ DESY, Notkestrae 85, D-22607 Hamburg, Germany \\ ${ }^{5}$ Center for Future High Energy Physics, Institute of High Energy Physics, \\ Chinese Academy of Sciences, Beijing 100049, China
}

(Received 21 December 2017; published 6 June 2018)

\begin{abstract}
We search for the fiveplet singly charged Higgs $H_{5}^{ \pm}$in the Georgi-Machacek model at the ep colliders. The charged Higgs bosons are produced via the $Z W^{ \pm}$fusion process $p e^{-} \rightarrow j e^{-} H_{5}^{ \pm}$and decay as $H_{5}^{ \pm} \rightarrow Z W^{ \pm} \rightarrow\left(l^{+} l^{-}\right)(j j)$. With a detector-level simulation at the FCC-eh and LHeC, a multivariate analysis is performed to yield limits on the production cross section times branching ratio $\sigma\left(p e^{-} \rightarrow j e^{-} H_{5}^{ \pm}\right) \times$ $\operatorname{BR}\left(H_{5}^{ \pm} \rightarrow Z W^{ \pm}\right)$and on the model parameter $\sin \theta_{H}$ for charged Higgs masses between 200 and $1000 \mathrm{GeV}$. Considering $10 \%$ systematic uncertainty on the background, at the FCC-eh (LHeC) with $1 \mathrm{ab}^{-1}$ integrated luminosity, for a $H_{5}^{ \pm}$mass of $600(200) \mathrm{GeV}$, the cross section corresponding to the 2- $\sigma$ significance is 0.59 (3.69) fb, corresponding to a $2-\sigma$ limit on the $\sin \theta_{H}$ is $0.15(0.41)$. The effects of $-80 \%$ and $+80 \%$ electron beam polarizations are also investigated and found to be small for this study.
\end{abstract}

DOI: 10.1103/PhysRevD.97.116005

\section{INTRODUCTION}

The discovery of the Higgs boson at the Large Hadron Collider (LHC) [1,2] is a major step towards an understanding of the electroweak symmetry breaking (EWSB) mechanism and marks a new era in particle physics, but this is not the end of the story. In fact, from a theoretical point of view, there is no fundamental reason for a minimal Higgs sector, as occurs in the Standard Model (SM). It is therefore important to consider extended scalar scenarios with higher isospin multiplets that may also contribute to EWSB. They could also provide a good way to generate a Majorana mass for neutrinos through the type-II seesaw [3] mechanism.

One such scenario is the Georgi-Machacek (GM) [4] model which contains a complex $S U(2)_{L}$ doublet field, a real triplet field, and a complex $S U(2)_{L}$ triplet field (see Sec. II for details). The exotic physical scalar states include a custodial fiveplet having neutral, singly, and doubly

\footnotetext{
*Corresponding author. kechen.wang@desy.de

Published by the American Physical Society under the terms of the Creative Commons Attribution 4.0 International license. Further distribution of this work must maintain attribution to the author(s) and the published article's title, journal citation, and DOI. Funded by SCOAP ${ }^{3}$.
}

charged members: $\mathrm{H}_{5}^{0}, \mathrm{H}_{5}^{ \pm}, \mathrm{H}_{5}^{ \pm \pm}$. Different charged Higgs boson states belonging to the same multiplet are expected to be degenerate in the GM model. Compared with the other Higgs extended models, such as a left-right symmetric model [5] or a little Higgs model [6], the GM model has some desirable features. It preserves the custodial $S U(2)_{C}$ symmetry at tree level, keeping the electroweak $\rho$ parameter equal to unity at tree level. It is thus less constrained experimentally [7]. We emphasize that in the GM model, the fiveplet $H_{5}$ is fermiophobic and the $H_{5}^{ \pm} W^{\mp} Z$ vertex appears at tree level. Since the charged Higgs members in 2-Higgs doublet models (2HDM) have only loop coupling to $\mathrm{WZ}$ [8] and since they couple to fermions, the measurement of the $H^{ \pm} W^{\mp} Z$ vertex can be used to distinguish models with singly charged Higgs bosons. It is also worth noting that the results of this study can be interpreted in terms of other models of an extended Higgs sector having a charged scalar [9-13].

Indirect limits on the GM model parameters can be set from B-physics and precision electroweak measurements, which set the upper limits on $\sin \theta_{H}$ linearly from 0.7 to 0.3 for the mass of the fiveplet states $M_{H_{5}}$ from 500 to $2000 \mathrm{GeV}$ and constrain the $\sin \theta_{H}$ below 0.7 for $M_{H_{5}}$ from 200 to $500 \mathrm{GeV}$ [14,15]. Direct limits on the parameter $\sin \theta_{H}$, based on the search for a heavy charged Higgs boson produced through vector boson fusion and decaying into $W Z$ bosons [16-18], have been obtained as a 
function of the charged Higgs mass by the ATLAS Collaboration from $p p$ collision at a center-of-mass energy of $8 \mathrm{TeV}$ [19]. Recently, more stringent limits have been reported by the CMS Collaboration from data at $\sqrt{s}=$ $13 \mathrm{TeV}$ with $15.2 \mathrm{fb}^{-1}$ luminosity [20]. The CMS Collaboration has reported limits on the parameter $\sin \theta_{H}$ [see Eq. (2)] as a function of mass for a doubly charged Higgs in the electroweak production of same-sign W boson pairs with a final stated consisting of two jets, two same-sign leptons, and missing energy, with $35.9 \mathrm{fb}^{-1}$ of integrated luminosity at a center-of-mass energy of $13 \mathrm{TeV}$ [21]. The sensitivity for the doubly charged Higgs searches at ep colliders has also been evaluated in Ref. [22].

In this paper, we evaluate the sensitivity of ep colliders to measure the vertex $H_{5}^{ \pm} W^{\mp} Z$. Based on the framework of the GM model, we perform a detector-level simulation at both the Large Hadron electron Collider (LHeC) and the electron-hadron mode of the Future Circular Collider (FCC-eh) for the signal process $p e^{-} \rightarrow j e^{-} H_{5}^{ \pm}$, produced via $W Z$ fusion and followed, with $100 \%$ branching ratio, by $H_{5}^{ \pm} \rightarrow Z W^{ \pm} \rightarrow\left(l^{+} l^{-}\right)(j j)$ (see Fig. 1). Other final state channels [such as $Z W \rightarrow(j j)(j j), \quad(j j)(l \nu), \quad(\nu \nu)(j j)$, $(\nu \nu)(l \nu),(l l)(l \nu)]$ can also be used to search for the $H_{5}^{ \pm}$. The fully hadronic final state $(j j)(j j)$ has the largest branching ratios but it suffers from poor mass reconstruction and combinatorial background. For the final states where $Z \rightarrow(\nu \nu)$, it is not possible to reconstruct the mass of the $Z$ boson. Therefore, although the semileptonic channel $Z W^{ \pm} \rightarrow\left(l^{+} l^{-}\right)(j j)$ has a relatively low branching ratio, it has a clean final state with a low SM background and a good reconstruction of the signal is made possible.

For the $e^{-}$and $p$ beam energies at the LHeC and FCC-eh colliders, we consider $60 \mathrm{GeV} \times 7 \mathrm{TeV}$ and $60 \mathrm{GeV} \times$ $50 \mathrm{TeV}[23,24]$, respectively. The maximal integrated luminosity for the $\mathrm{LHeC}$ is expected to be $1 \mathrm{ab}^{-1}$, while it can be $2.5 \mathrm{ab}^{-1}$ for the FCC-eh with a 25-year running $\left(1 \mathrm{ab}^{-1}\right.$ per 10 years).

Although the center-of-mass energies of 1.3 and $3.5 \mathrm{TeV}$, respectively, are lower than at $p p$ colliders, the SM QCD

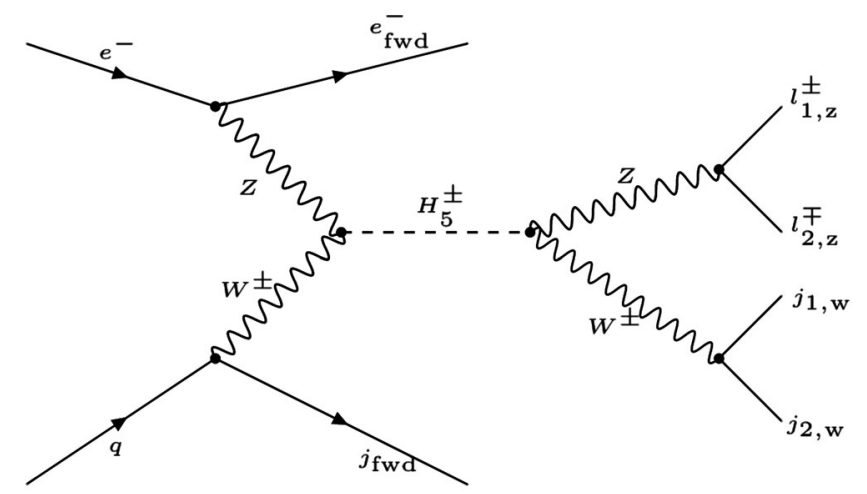

FIG. 1. The Feynman diagram for the signal production of $p e^{-} \rightarrow j e^{-} H_{5}^{ \pm}$, followed by the decays of $H_{5}^{ \pm} \rightarrow Z W^{ \pm} \rightarrow$ $\left(l^{+} l^{-}\right)(j j)$. backgrounds, which are dominant in the $p p$ vector boson fusion (VBF) process, are much smaller at ep colliders since there is no gluon exchange diagram. Furthermore, pileup jets, which can be misidentified as the forward jets in VBF, constitute an very important background at $p p$ colliders, especially at high luminosity, but they are essentially negligible at ep colliders.

The article is organized as follows. In Sec. II, we describe the GM model. In Sec. III, we discuss the distinguishing features of the signal and present the analysis method. In Sec. IV, we give the numerical results and set limits on the GM model parameters. We summarize and conclude in the last Sec. V.

\section{DESCRIPTION OF THE GEORGI-MACHACEK MODEL}

In the GM model, the Higgs sector consists of a complex $S U(2)_{L}$ isospin triplet $\left(\chi^{++}, \chi^{+}, \chi^{0}\right)$ with a hypercharge $\mathrm{Y}=1$, and a real triplet $\left(\xi^{+}, \xi^{0}, \xi^{-}\right)$with $\mathrm{Y}=0$ in addition to the original SM $S U(2)_{L}$ doublet $\left(\phi^{+}, \phi^{0}\right)$ (with $\mathrm{Y}=1 / 2)$. The scalar content of the theory can be organized in terms of a $S U(2)_{L} \otimes S U(2)_{R}$ symmetry. In order to make this symmetry explicit, the doublet is written in the form of a bidoublet $\Phi$ and the triplets are combined to form a bitriplet $\Delta$,

$\Phi=\left(\begin{array}{cc}\phi^{0 *} & \phi^{+} \\ \phi^{-} & \phi^{0}\end{array}\right), \quad \Delta=\left(\begin{array}{ccc}\chi^{0 *} & \xi^{+} & \chi^{++} \\ \chi^{-} & \xi^{0} & \chi^{+} \\ \chi^{--} & \xi^{-} & \chi^{0}\end{array}\right)$,

where we use the phase convention for the scalar field components: $\chi^{--}=\left(\chi^{++}\right)^{*}, \quad \chi^{-}=\left(\chi^{+}\right)^{*}, \quad \xi^{-}=\left(\xi^{+}\right)^{*}$, $\phi^{-}=\left(\phi^{+}\right)^{*}$.

After symmetry breaking, the scalar fields in the GM model can be classified into different representations under the custodial symmetry transformation. The doublet $\Phi$ is decomposed into a 3-plet and a singlet, and the triplet $\Delta$ is decomposed into a 5-plet, a 3-plet, and a singlet. Among these custodial multiplets, the 5-plet states are physical Higgs bosons, i.e., $H_{5}=\left(H_{5}^{ \pm \pm}, H_{5}^{ \pm}, H_{5}^{0}\right)$. A linear combinations of the two 3-plets corresponds to physical Higgs fields, i.e., $H_{3}=\left(H_{3}^{ \pm}, H_{3}^{0}\right)$, and the other becomes the Nambu-Goldstone bosons which are absorbed into the longitudinal components of the $W^{ \pm}$and $Z$ bosons, respectively. The Lagrangian and the full Feynman rules can be found, for example, in Ref. [25], where in our analysis, we concentrate on the study of $\mathrm{H}_{5}^{ \pm} \mathrm{W}^{\mp} \mathrm{Z}$ vertex. with the corresponding coupling

$$
g_{\mathrm{H}_{5}^{ \pm} \mathrm{W}^{\mp} \mathrm{Z}}=-\frac{e^{2} \mathrm{v}}{2 \cos \theta_{\mathrm{W}} \sin ^{2} \theta_{\mathrm{W}}} \sin \theta_{\mathrm{H}},
$$

where $\sin \theta_{\mathrm{H}} \equiv 2 \sqrt{2} \mathrm{v}_{\chi} / \mathrm{v}, \mathrm{v}_{\chi}$ is the vacuum expectation values of the $\chi$ field and $\mathrm{v}$ has the SM value of $246 \mathrm{GeV}$. 
Assuming a heavy $H_{3}$ multiplet, the $H_{5}$ scalars, which do not couple to fermions, will only be produced by vector boson fusion and will decay into a vector boson pair. The cross section and width are proportional to $\sin ^{2} \theta_{H}$.

\section{SEARCH STRATEGY}

The chain of data simulation starts with the event generator MADGRAPH5_AMC@NLO [26] using the nn23lo1 parton distribution function with a 4-flavor scheme. Parton showering and hadronization is then performed by Pythia [27]. Delphes [28] is used for detector simulation. The detector is assumed to have a cylindrical geometry comprising a central tracker followed by an electromagnetic and a hadronic calorimeter. The forward and backward regions are also covered by a tracker, an electromagnetic and a hadronic calorimeter. The angular acceptance for charged tracks in the pseudorapidity range of $-4.3<\eta<4.9$ and the detector performance in terms of momentum and energy resolution of electrons, muons, and jets, are based on the LHeC detector design [23,29]. For our simulation, a modified Pythia version tuned for the ep colliders and the Delphes card files for the $\mathrm{LHeC}$ and FCC-eh detector configurations [30] are used.

For the signal, we consider that only the 5-plet of the GM model is sufficiently light to be within reach of the collider while other new scalars are heavy and decoupled. The simplified model has only two free parameters: the mass of the 5-plet $M_{H_{5}^{ \pm}}$and the $\sin \theta_{H}$ value. The UFO model file from Ref. [31] is utilized to simulate the signal data.

Among the three leptons in the final state, one electron $e_{\text {fwd }}$ is from the incoming electron beam. The $\mathrm{Z}$ boson can be reconstructed from two opposite sign same flavor (OSSF) leptons $l^{+} l^{-}$(here $l$ denotes $e$ and $\mu$ only) which are ordered in decreasing transverse momentum and are here labeled as $l_{z, 1}$ and $l_{z, 2}$. Among the three jets, one forward jet $j_{\text {fwd }}$ is from the incoming proton beam. The leading and subleading jets from the $\mathrm{W}$ boson decay are labeled as $j_{\mathrm{w}, 1}$ and $j_{\mathrm{w}, 2}$.

In Fig. 2, we show the cross sections in fb at the FCC-eh and $\mathrm{LHeC}$, for the process $p e^{-} \rightarrow j e^{-} H_{5}^{ \pm}$followed by the decays of $H_{5}^{ \pm} \rightarrow Z W^{ \pm}$, as a function of the mass $M_{H_{5}^{ \pm}}$and with the model parameter $\sin \theta_{H}=0.5$. Another factor of $4.1 \%$ applies when considering the subsequent decays of $Z \rightarrow l^{+} l^{-}$and $W^{ \pm} \rightarrow j j$. It can be seen that at the FCC-eh, the $-80 \%(+80 \%)$ beam polarization increases (decreases) the production cross section by a factor of about $10 \%$, compared with the unpolarized beam case.

There are two main sources of SM background. The first source is the SM production of dibosons $Z V$, with the $Z$ boson decaying into two OSSF leptons, and another vector boson, $V=W^{ \pm}$or $Z$, decaying into two jets, i.e., $p e^{-} \rightarrow$ $j e^{-} Z V \rightarrow j e^{-}\left(l^{+} l^{-}\right)(j j)$. We label it as " $j e^{-} Z V^{\prime}$ " or "B 1 ". The second source is $Z+$ jets production, with the $Z$ boson decaying leptonically, and two jets produced from QCD radiation, i.e., $p e^{-} \rightarrow j e^{-} Z j j \rightarrow j e^{-}\left(l^{+} l^{-}\right) j j$. We label it as " $j e^{-} Z j j$ " or "B2". Compared with $j e^{-} Z j j, j e^{-} Z V$ is a

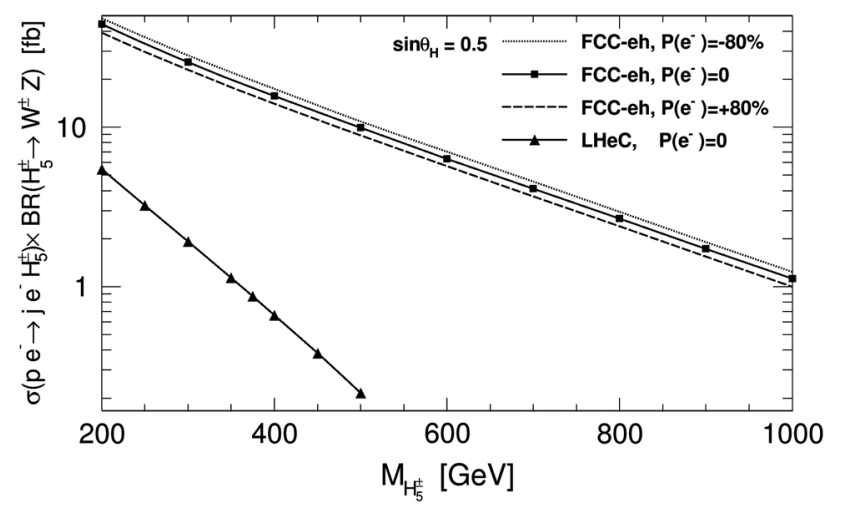

FIG. 2. The production cross section times branching ratio $\sigma\left(p e^{-} \rightarrow j e^{-} H_{5}^{ \pm}\right) \times \mathrm{BR}\left(H_{5}^{ \pm} \rightarrow Z W^{ \pm}\right)$in $\mathrm{fb}$ at the FCC-eh with $-80 \%$ polarized (dotted line), unpolarized (solid line with square markers), or $+80 \%$ polarized (dashed line) electron beam, and at the $\mathrm{LHeC}$ with unpolarized electron beam (solid line with triangle markers) as a function of the mass $M_{H_{5}^{ \pm}}$and assuming $\sin \theta_{H}=0.5$.

pure QED production, and its production cross section is consequently much smaller. However, since the two jets have the invariant mass of a $\mathrm{W}$ boson, it is an irreducible background. This is not the case for $j e^{-} Z j j$ which can therefore be suppressed more efficiently.

Other background processes with two OSSF leptons which do not have the invariant mass of the $Z$ boson may also contribute to the background but they can be effectively rejected and are hence neglected here.

For the event selection, we firstly apply the following preselection:

(1) At least three jets with $p_{T}>20 \mathrm{GeV}$;

(2) At least three leptons with $p_{T}>10 \mathrm{GeV}$; the charges and flavors of the leptons are required to be $\left(e^{-}, \mu^{+}, \mu^{-}\right)$or $\left(e^{-}, e^{+}, e^{-}\right)$;

(3) No b jet with $p_{T}>20 \mathrm{GeV}$.

In order to construct meaningful kinematical observables, the two leptons from the $\mathrm{Z}$ decay and the two jets from the $\mathrm{W}$ decay must be identified. If the OSSF pair of leptons is $\mu^{+} \mu^{-}$, there is no ambiguity. If there are two electrons in the final state, the OSSF pair $e^{+} e^{-}$with an invariant mass closest to the $Z$ boson mass will be identified as $l_{\mathrm{z}, 1}$ and $l_{\mathrm{z}, 2}$ and the third will be $e_{\mathrm{fwd}}$. Among the first three leading jets, the dijet pair with invariant mass closest to the $\mathrm{W}$ boson is considered to be $l_{\mathrm{w}, 1}$ and $l_{\mathrm{w}, 2}$, while the remaining jet is regarded as $j_{\text {fwd }}$.

After the preselection cuts and identification of $e_{\mathrm{fwd}}, l_{\mathrm{z}, 1}$, $l_{\mathrm{z}, 2}$, and $j_{\mathrm{fwd}}, l_{\mathrm{w}, 1}, l_{\mathrm{w}, 2}$, the following 34 kinematical observables are reconstructed and input into the TMVA package [32] to perform the boosted decision trees (BDT) analysis.

(1) global observables:

(a) the missing energy $\mathbb{E}_{T}$;

(b) the scalar sum of the transverse momentum $p_{T}$ of all jets $H_{T}$. 
(2) observables for the forward objects:

(a) $p_{T}$ and the pseudorapidity $\eta$ of $e_{\text {fwd }}$ and $j_{\text {fwd }}$ : $p_{T}\left(e_{\mathrm{fwd}}\right), \eta\left(e_{\mathrm{fwd}}\right), p_{T}\left(j_{\mathrm{fwd}}\right), \eta\left(j_{\mathrm{fwd}}\right)$;

(b) $p_{T}, \eta$, and invariant mass $M$ of the system of $e_{\mathrm{fwd}}$ and $j_{\text {fwd }}: p_{T}\left(e_{\text {fwd }}+j_{\text {fwd }}\right), \eta\left(e_{\text {fwd }}+j_{\text {fwd }}\right)$, and $M\left(e_{\mathrm{fwd}}+j_{\mathrm{fwd}}\right)$

(c) the pseudorapidity difference $\Delta \eta$ and the azimuthal angle difference $\Delta \phi$ between $e_{\mathrm{fwd}}$ and $j_{\mathrm{fwd}}$ : $\Delta \eta\left(e_{\mathrm{fwd}}, j_{\mathrm{fwd}}\right), \Delta \phi\left(e_{\mathrm{fwd}}, j_{\mathrm{fwd}}\right)$.

(3) observables for the final state $Z$ system:

(a) $p_{T}$ and $\eta$ of $l_{z, 1}$ and $l_{z, 2}: p_{T}\left(l_{z, 1}\right), \eta\left(l_{z, 1}\right)$, $p_{T}\left(l_{\mathrm{z}, 2}\right), \eta\left(l_{\mathrm{z}, 2}\right)$

(b) $p_{T}, \eta$, and $M$ of the system of $l_{\mathrm{z}, 1}$ and $l_{\mathrm{z}, 2}$ : $p_{T}\left(l_{\mathrm{z}, 1}+l_{\mathrm{z}, 2}\right), \eta\left(l_{\mathrm{z}, 1}+l_{\mathrm{z}, 2}\right)$, and $M\left(l_{\mathrm{z}, 1}+l_{\mathrm{z}, 2}\right)$;

(c) $\Delta \eta$ and $\Delta \phi$ between $l_{z, 1}$ and $l_{\mathrm{z}, 2}$ : $\Delta \eta\left(l_{\mathrm{z}, 1}, l_{\mathrm{z}, 2}\right), \Delta \phi\left(l_{\mathrm{z}, 1}, l_{\mathrm{z}, 2}\right)$.

(4) observables for the final state $W$ system:

(a) $p_{T}$ and $\eta$ of $j_{\mathrm{w}, 1}$ and $j_{\mathrm{w}, 2}: p_{T}\left(j_{\mathrm{w}, 1}\right), \eta\left(j_{\mathrm{w}, 1}\right)$, $p_{T}\left(j_{\mathrm{w}, 2}\right), \eta\left(j_{\mathrm{w}, 2}\right)$
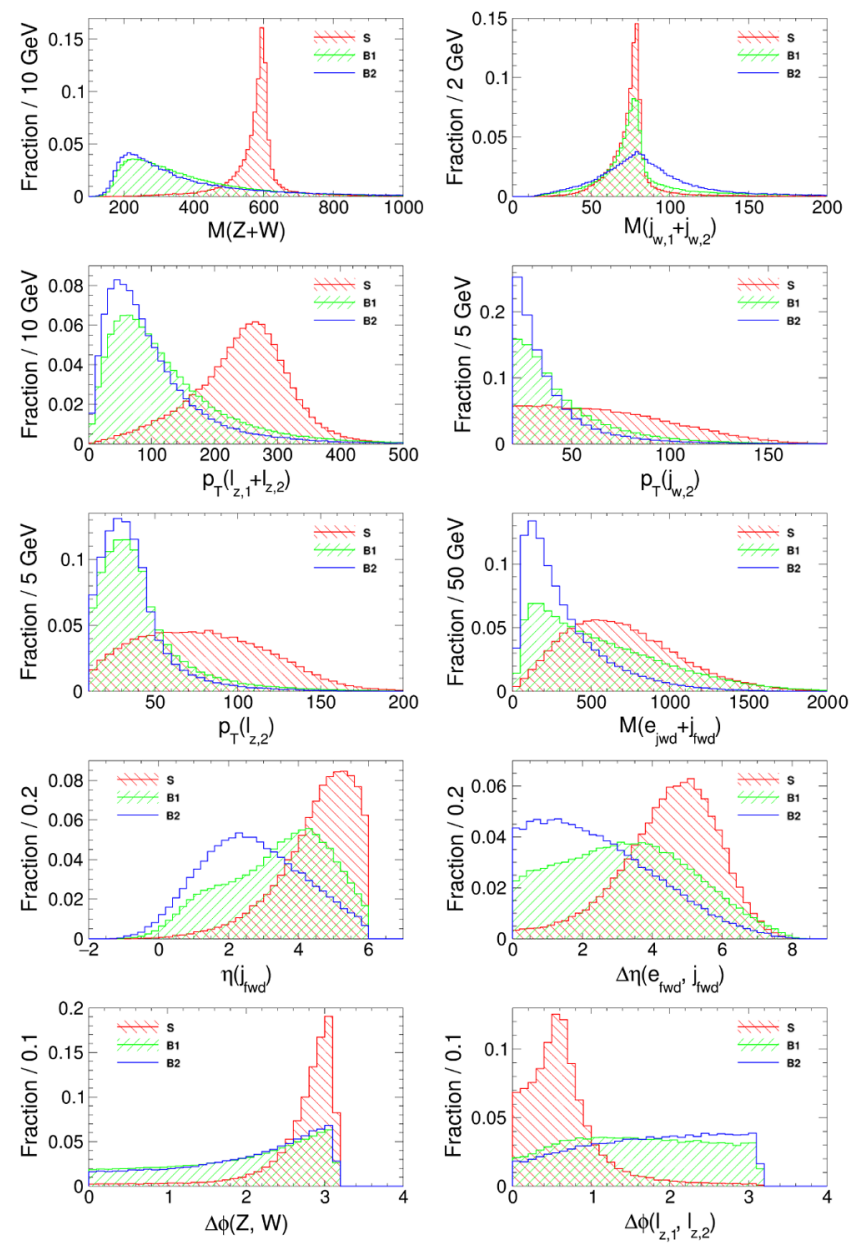

FIG. 3. Kinematical distributions of some input observables for signal (S, red) with benchmark $M_{H_{5}^{ \pm}}=600 \mathrm{GeV}$, and for the SM background $j e^{-} Z V$ (B1, green) and $j e^{-} Z j j$ (B2, blue) after applying the preselection cuts at the FCC-eh with the unpolarized electron beam. (b) $p_{T}, \eta$, and $M$ of the system of $j_{\mathrm{w}, 1}$ and $j_{\mathrm{w}, 2}$ : $p_{T}\left(j_{\mathrm{w}, 1}+j_{\mathrm{w}, 2}\right), \eta\left(j_{\mathrm{w}, 1}+j_{\mathrm{w}, 2}\right)$, and $M\left(j_{\mathrm{w}, 1}+j_{\mathrm{w}, 2}\right)$;

(c) $\Delta \eta$ and $\Delta \phi$ between $j_{\mathrm{w}, 1}$ and $j_{\mathrm{w}, 2}$ : $\Delta \eta\left(j_{\mathrm{w}, 1}, j_{\mathrm{w}, 2}\right), \Delta \phi\left(j_{\mathrm{w}, 1}, j_{\mathrm{w}, 2}\right)$.

(5) observables for the final state $Z+W$ system:

(a) $p_{T}, \eta$, and $M$ of the system of the reconstructed $\mathrm{W}$ and $\mathrm{Z}$ bosons: $p_{T}(Z+W), \eta(Z+W)$, and $M(Z+W)$

(b) $\Delta \eta$ and $\Delta \phi$ between the reconstructed $\mathrm{W}$ and $\mathrm{Z}$ bosons: $\Delta \eta(Z, W), \Delta \phi(Z, W)$.

Figure 3 shows the kinematical distributions of some input observables at the FCC-eh with an unpolarized electron beam for the signal benchmark point with $M_{H_{5}^{ \pm}}=600 \mathrm{GeV}$ (S, red) and for the SM backgrounds $j e^{-} Z V$ (B1, green) and $j e^{-} Z j j(\mathrm{~B} 2$, blue) after applying the preselection cuts. From the $M(Z+W)$ plot, one can see clearly that the charged Higgs mass is reconstructed with good resolution, peaking sharply around the benchmark mass $600 \mathrm{GeV}$. The separation between the signal and background is quite good for all masses tested. Therefore, $M(Z+W)$ is a useful and important variable when searching for a resonance at a specific mass. Since the kinematical distributions change as a function of the charged Higgs mass, the mass is scanned from $200 \mathrm{GeV}$ to $1 \mathrm{TeV}$ in steps of $100 \mathrm{GeV}$ and the BDT analysis is reoptimized in each case.

\section{RESULTS}

The observables are input to the TMVA package to perform the BDT analysis. For the benchmark point $M_{H_{5}^{ \pm}}=600 \mathrm{GeV}$, the training and test process reveals that the most useful observables in order of their ranking are $M(Z+W), \eta\left(j_{\mathrm{fwd}}\right), p_{T}\left(l_{\mathrm{z}, 1}+l_{\mathrm{z}, 2}\right), \eta\left(e_{\mathrm{fwd}}\right), \Delta \phi\left(l_{\mathrm{z}, 1}, l_{\mathrm{z}, 2}\right)$, $\Delta \eta\left(e_{\mathrm{fwd}}, j_{\mathrm{fwd}}\right), M\left(j_{\mathrm{w}, 1}+j_{\mathrm{w}, 2}\right), \eta\left(l_{\mathrm{z}, 2}\right), \eta\left(j_{\mathrm{w}, 1}\right), p_{T}\left(j_{\mathrm{w}, 2}\right)$, $\eta\left(l_{\mathrm{z}, 1}\right), p_{T}\left(j_{\mathrm{fwd}}\right)$.

It is worth noting that the BDT analysis, based on the multivariant method, can account for the correlations between the input observables and optimize the selection, leading usually to better limits compared to a traditional "cut-and-count" analysis. Since we used 34 kinematical observables, it would be almost impossible for a "cut-andcount" analysis to take into account their correlations and find the optimized cuts for all of them. Other automatic optimization methods, like the neural network method, are expected to have a similar efficiency to the BDT method.

In Fig. 4, we show the distribution of the BDT response for the signal benchmark point with $M_{H_{5}^{ \pm}}=600 \mathrm{GeV}$ (S, red) and for the SM background $j e^{-} Z V$ (B1, green) and $j e^{-} Z j j$ (B2, blue). A cut on the BDT response is chosen to maximize the signal significance. Assuming an integrated luminosity of $1 \mathrm{ab}^{-1}$ at the FCC-eh (LHeC) with unpolarized electron beam, the cut-flow Table I shows the number of events remaining at different stages of the analysis for the signal with $M_{H_{5}^{ \pm}}=600$ (200) GeV and 


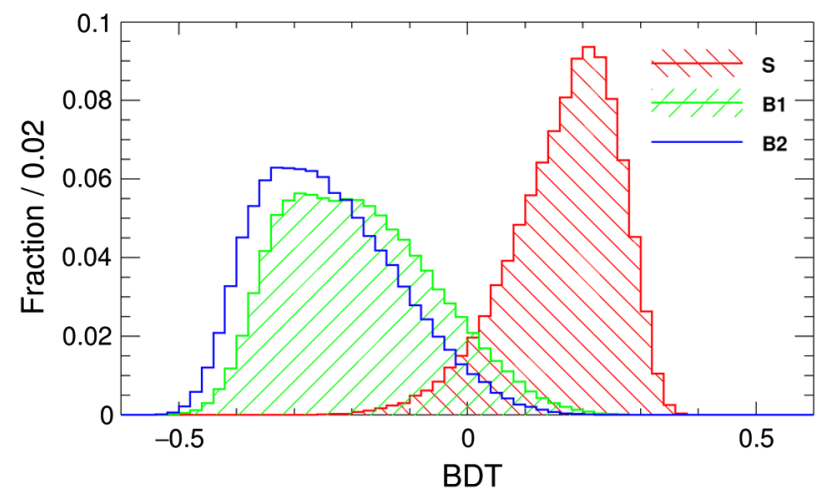

FIG. 4. Distribution of BDT response for the signal (S, red) with benchmark $M_{H_{5}^{ \pm}}=600 \mathrm{GeV}$, and for the SM background $j e^{-} Z V$ (B1, green) and $j e^{-} Z j j$ (B2, blue) after applying the preselection cuts at the $\mathrm{FCC}$-eh with the unpolarized electron beam.

$\sin \theta_{H}=0.5$, and for the backgrounds $j e^{-} Z V$ and $j e^{-} Z j j$. The signal to background ratio, after selection, is excellent $(\sim 14)$ at the FCC-eh, demonstrating the importance of ep colliders since, in the case of the CMS measurement of $H_{5}^{ \pm}$at the LHC, in the signal region around the peak the signal to background ratio is closer to $\sim 1$ [20].

The signal significance, $\sigma_{\text {stat }}$, of the potential signal is evaluated as

$$
\sigma_{\text {stat }}=\sqrt{2\left[\left(N_{s}+N_{b}\right) \ln \left(1+\frac{N_{s}}{N_{b}}\right)-N_{s}\right]},
$$

where $N_{s}\left(N_{b}\right)$ are the expected number of events for signal (background).

Taking into account a systematic uncertainty of $\sigma_{b}$ in the evaluation of the number of background events, Eq. (4) will be used to evaluate the significance,

TABLE I. Cut-flow table at the FCC-eh (LHeC) for the signal (S) benchmark point with $M_{H_{5}^{ \pm}}=600$ (200) $\mathrm{GeV}$ and $\sin \theta_{H}=0.5$, and the SM background $j e^{-} Z V$ (B1) and $j e^{-} Z j j$ (B2). The numbers of events correspond to an integrated luminosity of $1 \mathrm{ab}^{-1}$ with unpolarized electron beam. The optimized BDT cut is BDT $>0.189(0.119)$ for the FCC-eh $(\mathrm{LHeC})$. The signal significances with $0 \%$ and $10 \%$ systematic uncertainty on background are presented in the last two rows.

\begin{tabular}{lccccccc}
\hline \hline & \multicolumn{3}{c}{ FCC-eh } & & \multicolumn{3}{c}{ LHeC } \\
\cline { 2 - 4 } \cline { 5 - 7 } Cuts & $\mathrm{S}$ & $\mathrm{B} 1$ & $\mathrm{~B} 2$ & & $\mathrm{~S}$ & $\mathrm{~B} 1$ & $\mathrm{~B} 2$ \\
\hline Initial & 260 & $1.09 \times 10^{4}$ & $1.52 \times 10^{5}$ & & 220 & 531 & $3.19 \times 10^{4}$ \\
Preselection & 102 & 751 & 6442 & & 13 & 11 & 148 \\
BDT & 47.7 & 1.7 & 1.7 & & 4.9 & 0.2 & 0.8 \\
$\sigma_{\text {stat }}$ & & 13.5 & & & 3.35 & \\
$\sigma_{\text {stat }+10 \% \text { syst }}$ & \multicolumn{4}{c}{12.7} & & & 3.32 \\
\hline \hline
\end{tabular}

$$
\begin{aligned}
\sigma_{\text {stat }+ \text { syst }}= & {\left[2 \left(\left(N_{s}+N_{b}\right) \ln \frac{\left(N_{s}+N_{b}\right)\left(N_{b}+\sigma_{b}^{2}\right)}{N_{b}^{2}+\left(N_{s}+N_{b}\right) \sigma_{b}^{2}}\right.\right.} \\
& \left.\left.-\frac{N_{b}^{2}}{\sigma_{b}^{2}} \ln \left(1+\frac{\sigma_{b}^{2} N_{s}}{N_{b}\left(N_{b}+\sigma_{b}^{2}\right)}\right)\right)\right]^{1 / 2}
\end{aligned}
$$

For the signal benchmark point at the FCC-eh, the statistical significance is found to be $13.5 \sigma$. It is difficult to estimate the systematic uncertainties on the background. However, since the signal is well reconstructed as a narrow resonance over a smooth background, a data driven method using the sideband distributions can be used to constrain the level of background. Assuming a systematic uncertainty of $\sigma_{b}=10 \% \times N_{b}$ on the background, the signal significance reduces to $12.7 \sigma$.

Since the production cross section $\sigma\left(p e^{-} \rightarrow j e^{-} H_{5}^{ \pm}\right)$is proportional to $\sin ^{2} \theta_{H}$, these results can be reinterpreted in terms of limits on the parameter $\sin \theta_{H}$. For the benchmark point of $M_{H_{5}^{ \pm}}=600 \mathrm{GeV}$ at the FCC-eh, the $\sin \theta_{H}$ values corresponding to significances of $5 \sigma, 3 \sigma$, and $2 \sigma$, assuming $10 \%$ systematic uncertainty on the background, are found to be $0.26,0.19$, and 0.15 , respectively.

The upper plot of Fig. 5 shows, as a function of $M_{H_{5}^{ \pm}}$, the cross section times branching ratio $\sigma\left(p e^{-} \rightarrow j e^{-} H_{5}^{ \pm}\right) \times$ $\operatorname{BR}\left(H_{5}^{ \pm} \rightarrow Z W^{ \pm}\right)$which can lead to an observation with
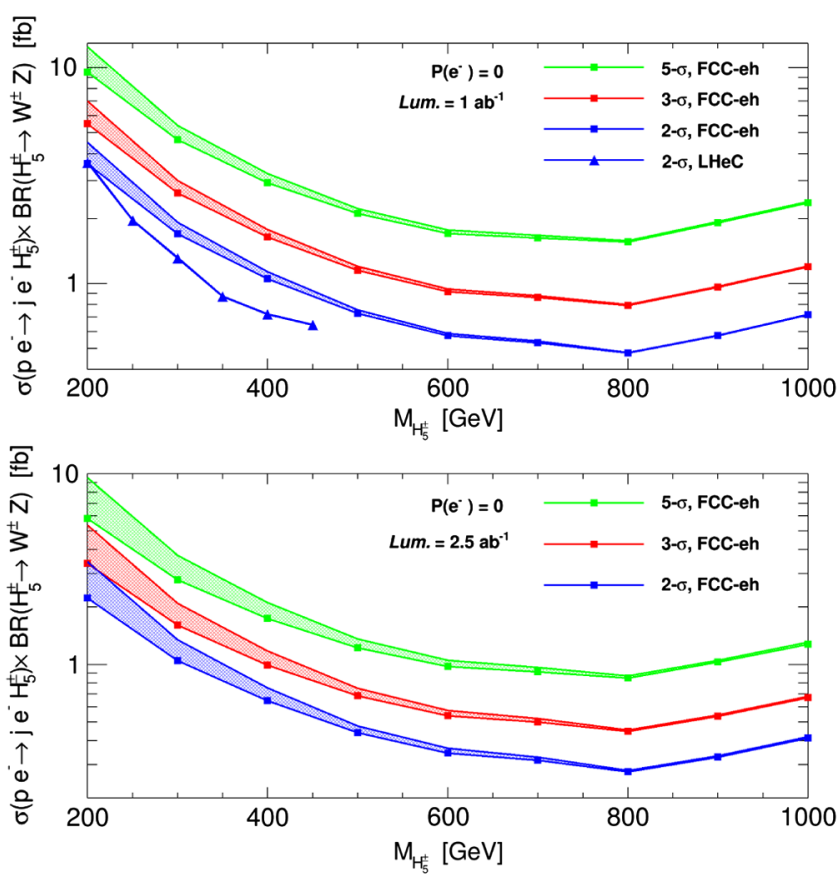

FIG. 5. The significance contour bands in the plane of production cross section times branching ratio $\sigma\left(p e^{-} \rightarrow j e^{-} H_{5}^{ \pm}\right) \times$ $\mathrm{BR}\left(H_{5}^{ \pm} \rightarrow Z W^{ \pm}\right)$vs $M_{H_{5}^{ \pm}}$for the FCC-eh and LHeC with unpolarized electron beams and luminosity of $1 \mathrm{ab}^{-1}$ (upper plot) and for the FCC-eh with unpolarized electron beams and luminosity of $2.5 \mathrm{ab}^{-1}$ (lower plot). For each band, the bottom (top) of the shaded region denotes the significance curve with $0 \%$ $(10 \%)$ systematic uncertainty on the background. 
significance of $2 \sigma, 3 \sigma$, and $5 \sigma$, assuming $1 \mathrm{ab}^{-1}$ of integrated luminosity at the FCC-eh. The $2 \sigma$ sensitivity at the $\mathrm{LHeC}$ is shown on the same plot, even though the center-of-mass energy of the collider is different. For the heavy mass points at the FCC-eh and for all the mass points at the $\mathrm{LHeC}$, due to the low number of background events expected after the final BDT cut, a $10 \%$ systematic uncertainty on the background has a negligible effect on the sensitivity of the measurement. At the LHeC with $10 \%$ systematic uncertainty on the background, for the $200 \mathrm{GeV}$ benchmark point, the cross sections corresponding to the $2-\sigma$ significance is $3.69 \mathrm{fb}$. The lower plot shows the same plot for FCC-eh with an integrated luminosity of $2.5 \mathrm{ab}^{-1}$. For the benchmark $600 \mathrm{GeV}$ point at the FCC-eh with 1 (2.5) $\mathrm{ab}^{-1}$ luminosity, considering $10 \%$ systematic uncertainty on the background, the cross sections corresponding to the $2,3,5-\sigma$ significances are $0.59(0.36), 0.95(0.58)$, $1.78(1.05) \mathrm{fb}$, respectively.

Figure 6 shows the significance contour bands in the plane of $\sin \theta_{H}$ vs the fiveplet mass $M_{H_{5}}$ for the FCC-eh and $\mathrm{LHeC}$ with unpolarized electron beams and luminosity of $1 \mathrm{ab}^{-1}$ (upper plot) and for the FCC-eh with unpolarized
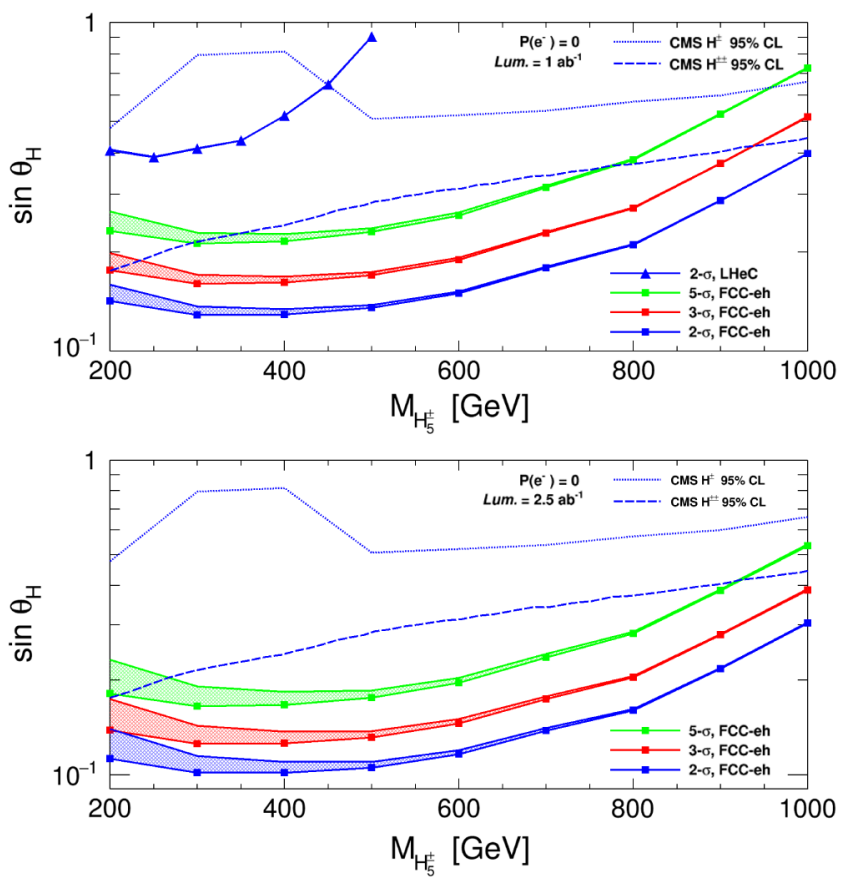

FIG. 6. The significance contour bands in the plane of $\sin \theta_{H}$ vs $M_{H_{5}}$ for the FCC-eh and $\mathrm{LHeC}$ with unpolarized electron beams and luminosity of $1 \mathrm{ab}^{-1}$ (upper plot) and for the FCC-eh with unpolarized electron beams and luminosity of $2.5 \mathrm{ab}^{-1}$ (lower plot). For each band, the bottom (top) of the shaded region denotes the significance curve with $0 \%$ (10\%) systematic uncertainty on the background. The blue dotted curve gives the $95 \%$ C.L. limit on the singly charged $H_{5}^{ \pm}$searches at the CMS from Ref. [20], while the blue dashed curve denotes the 95\% C.L. limit on the doubly charged $H_{5}^{ \pm \pm}$searches at the CMS from Ref. [21]. electron beams and luminosity of $2.5 \mathrm{ab}^{-1}$ (lower plot). Also shown are the current $95 \%$ C.L. limits on the singly charged $H_{5}^{ \pm}$searches [20] and on the doubly charged $H_{5}^{ \pm \pm}$ searches [21] obtained by the CMS Collaboration. At the FCC-eh with $10 \%$ systematic uncertainty on the background and $1 \mathrm{ab}^{-1}$ luminosity, the 2 (5)- $\sigma$ limits on the model parameter $\sin \theta_{H}$ are found to be $0.15(0.26)$ for the benchmark $600 \mathrm{GeV}$ mass. With $2.5 \mathrm{ab}^{-1}$ luminosity, the limits can be reduced to $0.12(0.20)$. For the benchmark $200 \mathrm{GeV}$ mass point at the $\mathrm{LHeC}$, with $10 \%$ systematic uncertainty on the background and $1 \mathrm{ab}^{-1}$ luminosity, the 2- $\sigma$ limits on the $\sin \theta_{H}$ is 0.41 . Comparing the $1 \mathrm{ab}^{-1}$ luminosity limits at the $\mathrm{LHeC}$ and FCC-eh with the current CMS limits from the singly charged Higgs searches, based on $15.2 \mathrm{fb}^{-1}$ of data at $13 \mathrm{TeV}$, the LHeC $2-\sigma$ limits are still stronger for the lower masses, while the FCC-eh 2- $\sigma$ limits are much stronger for all masses. The current doubly charged Higgs searches by CMS, based on $35.9 \mathrm{fb}^{-1}$ of data at $13 \mathrm{TeV}$, obtain similar limits for $200 \mathrm{GeV}$ and for $1000 \mathrm{GeV}$ masses to those of the FCC-eh. However, the CMS limits are much weaker for masses around $500 \mathrm{GeV}$. The FCC-eh limits with $2.5 \mathrm{ab}^{-1}$ luminosity are stronger than the current CMS limits from doubly charged Higgs searches for all masses. We have assumed degenerate masses for $H_{5}^{ \pm \pm}$and $H_{5}^{ \pm}$here, which may not be the case in a more generic model. Finally, no predictions are available for the high luminosity LHC future collider, but it can be expected that the pileup background would be more important than at the LHC.

As shown in the Fig. 2, at the FCC-eh for a given mass, a $-80 \%(+80 \%)$ polarization of electron beam increases (decreases) the production cross section of the signal by a factor of about $10 \%$ compared with the case of an unpolarized beam. It is found that with the same beam polarizations the cross section of backgrounds $j e^{-} Z V$ and $j e^{-} Z j j$ will also increase (decrease) by factors of about $10 \%$ and $25 \%$, respectively. Moreover, we find that the kinematical distributions of some input observables, such as $\eta\left(e_{\text {fwd }}\right)$, $p_{T}\left(e_{\mathrm{fwd}}\right), \Delta \eta\left(e_{\mathrm{fwd}}, j_{\mathrm{fwd}}\right), \Delta \phi\left(e_{\mathrm{fwd}}, j_{\mathrm{fwd}}\right), p_{T}\left(e_{\mathrm{fwd}}+j_{\mathrm{fwd}}\right)$, are quite different in the two cases. It is therefore not possible to simply scale the cross sections to infer the limits with polarized beams. For the benchmark $M_{H_{5}^{ \pm}}=600 \mathrm{GeV}$, after performing the full analysis with simulation of both the signal and background data in the polarized electron beam cases, we find at the FCC-eh with $1 \mathrm{ab}^{-1}$ luminosity, the 2- $\sigma$ limits on the $\sin \theta_{H}$ change only from 0.152 in the case of unpolarized beam to $0.157(0.148)$ in the cases of $-80 \%$ $(+80 \%)$ polarization. Therefore, beam polarization has a very limited effect on the sensitivity of signal for this study.

\section{CONCLUSIONS}

We develop the search strategy for the singly charged 5-plet Higgs in the Georgi-Machacek model at the ep colliders. The charged Higgs are produced by the vector boson fusion process, $p e^{-} \rightarrow j e^{-} H_{5}^{ \pm}$, and followed by the 
decays of $H_{5}^{ \pm} \rightarrow Z W^{ \pm} \rightarrow\left(l^{+} l^{-}\right)(j j)$. With a detector simulation, we adopt the BDT method to perform the multivariate analysis and extract the potential signal from the background. Assuming 10\% uncertainty on the background, at the FCC-eh with an unpolarized electron beam and an integrated luminosity of 1 and $2.5 \mathrm{ab}^{-1}$, we find the 2,3 , and 5- $\sigma$ limits on the production cross section times branching ratio $\sigma\left(p e^{-} \rightarrow j e^{-} H_{5}^{ \pm}\right) \times \mathrm{BR}\left(H_{5}^{ \pm} \rightarrow Z W^{ \pm}\right)$and on the model parameter $\sin \theta_{H}$ for charged Higgs masses in the range $200-1000 \mathrm{GeV}$. The $2-\sigma$ limits at the $\mathrm{LHeC}$ with $1 \mathrm{ab}^{-1}$ luminosity are also presented. The effects of electron beam polarization are investigated and found to be small for this study.

Compared with the present limits obtained from the singly charged Higgs searches at the LHC, the LHeC center-of-mass energy does not allow a competitive test of the GM model, while the FCC-eh limits are much stronger for all masses. The FCC-eh has therefore a very good potential to search for the charged Higgs with coupling to the vector bosons and for testing the GM model.

\section{ACKNOWLEDGMENTS}

We thank Max Klein, Uta Klein, Peter Kostka, Satoshi Kawaguchi, Masahiro Kuze, and Monica D'Onofrio for the data simulation and helpful communications. We appreciate the comments from other members in the LHeC FCCeh Higgs and BSM physics study groups. K. W. also wants to thank Christophe Grojean for helpful discussions and supports. H.S. is supported by the National Natural Science Foundation of China (Grant No. 11675033) and by the Fundamental Research Funds for the Central Universities (Grant No. DUT18LK27); K. W. by the International Postdoctoral Exchange Fellowship Program (No. 90 Document of OCPC, 2015); G. A. by National Science and Engineering Research Council (Canada).
[1] G. Aad et al. (ATLAS Collaboration), Observation of a new particle in the search for the Standard Model Higgs boson with the ATLAS detector at the LHC, Phys. Lett. B 716, 1 (2012).

[2] S. Chatrchyan et al. (CMS Collaboration), Observation of a new boson at a mass of $125 \mathrm{GeV}$ with the CMS experiment at the LHC, Phys. Lett. B 716, 30 (2012).

[3] T. P. Cheng and L. F. Li, Neutrino masses, mixings and oscillations in $\mathrm{SU}(2) \times \mathrm{U}(1)$ models of electroweak interactions, Phys. Rev. D 22, 2860 (1980); J. Schechter and J. W. F. Valle, Neutrino masses in $\mathrm{SU}(2) \times \mathrm{U}(1)$ theories, Phys. Rev. D 22, 2227 (1980); G. Lazarides, Q. Shafi, and C. Wetterich, Proton lifetime and fermion masses in an SO (10) model, Nucl. Phys. B181, 287 (1981); R. N. Mohapatra and G. Senjanovic, Neutrino masses and mixings in gauge models with spontaneous parity violation, Phys. Rev. D 23, 165 (1981); M. Magg and C. Wetterich, Neutrino mass problem and gauge hierarchy, Phys. Lett. 94B, 61 (1980).

[4] H. Georgi and M. Machacek, Doubly charged Higgs bosons, Nucl. Phys. B262, 463 (1985); M. S. Chanowitz and M. Golden, Higgs boson triplets with $M_{W}=M_{Z} \cos \theta \omega$, Phys. Lett. 165B, 105 (1985).

[5] R. N. Mohapatra and J. C. Pati, A natural left right symmetry, Phys. Rev. D 11, 2558 (1975); G. Senjanovic and R. N. Mohapatra, Exact left right symmetry and spontaneous violation of parity, Phys. Rev. D 12, 1502 (1975).

[6] N. Arkani-Hamed, A. G. Cohen, E. Katz, and A. E. Nelson, The littlest Higgs, J. High Energy Phys. 07 (2002) 034.

[7] S. Godfrey and K. Moats, Exploring Higgs triplet models via vector boson scattering at the LHC, Phys. Rev. D 81, 075026 (2010).

[8] J. A. Grifols and A. Mendez, $W Z H^{ \pm}$coupling in $S U(2) \times$ $U(1)$ gauge models, Phys. Rev. D 22, 1725 (1980).
[9] J. L. Diaz-Cruz, J. Hernandez-Sanchez, and J. J. Toscano, An effective Lagrangian description of charged Higgs decays $H^{+} \rightarrow W^{+} \gamma, W^{+} Z$ and $W^{+} h^{0}$, Phys. Lett. B 512, 339 (2001).

[10] K. Cheung and D. K. Ghosh, Triplet Higgs boson at hadron colliders, J. High Energy Phys. 11 (2002) 048.

[11] G. C. Branco, P. M. Ferreira, L. Lavoura, M. N. Rebelo, M. Sher, and J. P. Silva, Theory and phenomenology of twoHiggs-doublet models, Phys. Rep. 516, 1 (2012).

[12] D. de Florian et al. (LHC Higgs Cross Section Working Group), Handbook of LHC Higgs Cross Sections: 4. Deciphering the Nature of the Higgs Sector, arXiv:1610.07922.

[13] I. P. Ivanov, Building and testing models with extended Higgs sectors, Prog. Part. Nucl. Phys. 95, 160 (2017).

[14] M. Zaro and H. Logan, Recommendations for the interpretation of LHC searches for $H_{5}^{0}, H_{5}^{ \pm}$, and $H_{5}^{ \pm \pm}$in vector boson fusion with decays to vector boson pairs, http://cds .cern.ch/record/2002500, [LHCHXSWG-2015-001].

[15] K. Hartling, K. Kumar, and H. E. Logan, Indirect constraints on the Georgi-Machacek model and implications for Higgs boson couplings, Phys. Rev. D 91, 015013 (2015).

[16] C. W. Chiang and K. Tsumura, Properties and searches of the exotic neutral Higgs bosons in the Georgi-Machacek model, J. High Energy Phys. 04 (2015) 113.

[17] Y. Zhang, H. Sun, X. Luo, and W. Zhang, Searching for the heavy charged custodial fiveplet Higgs boson in the GeorgiMachacek model at the International Linear Collider, Phys. Rev. D 95, 115022 (2017).

[18] S. Kanemura, K. Yagyu, and K. Yanase, Testing Higgs models via the $H^{ \pm} W^{\mp} Z$ vertex by a recoil method at the International Linear Collider, Phys. Rev. D 83, 075018 (2011).

[19] G. Aad et al. (ATLAS Collaboration), Search for a Charged Higgs Boson Produced in the Vector-boson Fusion Mode with 
Decay $H \pm$ to $W^{ \pm} Z$ using pp Collisions at $\sqrt{s}=8 \mathrm{TeV}$ with the ATLAS Experiment, Phys. Rev. Lett. 114, 231801 (2015).

[20] A. M. Sirunyan et al. (CMS Collaboration), Search for Charged Higgs Bosons Produced via Vector Boson Fusion and Decaying into a Pair of $W$ and $Z$ Bosons Using $p p$ Collisions at $\sqrt{s}=13 \mathrm{TeV}$, Phys. Rev. Lett. 119, 141802 (2017).

[21] A. M. Sirunyan et al. (CMS Collaboration), Observation of Electroweak Production of Same-Sign W Boson Pairs in the Two Jet and Two Same-Sign Lepton Final State in ProtonProton Collisions at $\sqrt{s}=13 \mathrm{TeV}$, Phys. Rev. Lett. 120, 081801 (2018).

[22] H. Sun, X. Luo, W. Wei, and T. Liu, Searching for the doublycharged Higgs bosons in the Georgi-Machacek model at the electron-proton colliders, Phys. Rev. D 96, 095003 (2017).

[23] J. L. Abelleira Fernandez et al. (LHeC Study Group), A large hadron electron collider at CERN: Report on the physics and design concepts for machine and detector, J. Phys. G 39, 075001 (2012).

[24] O. Brüning et al., Future circular collider study FCC-he baseline parameters, https://fcc.web.cern.ch/Documents/ FCCheBaselineParameters.pdf.

[25] K. Hartling, K. Kumar, and H. E. Logan, The decoupling limit in the Georgi-Machacek model, Phys. Rev. D 90, 015007 (2014).
[26] J. Alwall, R. Frederix, S. Frixione, V. Hirschi, F. Maltoni, O. Mattelaer, H.-S. Shao, T. Stelzer, P. Torrielli, and M. Zaro, The automated computation of tree-level and next-toleading order differential cross sections, and their matching to parton shower simulations, J. High Energy Phys. 07 (2014) 79 .

[27] T. Sjostrand, S. Mrenna, and P. Skands, A brief Introduction to PYTHIA8.1, Comput. Phys. Commun. 178, 852 (2008).

[28] J. de Favereau, C. Delaere, P. Demin, A. Giammanco, V. Lemaître, A. Mertens, and M. Selvaggi (DELPHES 3 Collaboration), DELPHES 3, A modular framework for fast simulation of a generic collider experiment, J. High Energy Phys. 02 (2014) 057.

[29] M. Klein, in LHeC Detector Design, 25th International Workshop on Deep Inelastic Scattering, Birmingham, 2017, https://indico.cern.ch/event/568360/contributions/2523637/.

[30] M. Klein, U. Klein, P. Kostka, and S. Kawaguchi (private communication).

[31] K. Kumar, K. Hartling, and H. Logan, GeorgiMachacek Model, http://feynrules.irmp.ucl.ac.be/wiki/ GeorgiMachacekModel.

[32] A. Hoecker, P. Speckmayer, J. Stelzer, J. Therhaag, E. von Toerne, and H. Voss, TMVA: Toolkit for multivariate data analysis, Proc. Sci., ACAT2007 (2007) 040. 